\title{
QoS Evaluation Based on Fuzzy Neural Networks
}

\author{
Khurrum Jalil a, Khurrum Hussain ${ }^{b}$, Asad Ahmad ${ }^{\text {c}}$, Xiaolin Gui ${ }^{\mathrm{d}}$ \\ School of Electronics and Information Engineering, Xi'an Jiaotong University, Xian 710049, China \\ akhurrumjalil@gmail.com, bzakirkhurram18@gmail.com, cgeminiasad66@yahoo.com, \\ dguixjtu@126.com
}

\begin{abstract}
In order to improve the Quality of service (QoS) and the Quantity of user Experience, overlay network visualized the network application and underlay structure. An algorithm named MOO-GSON is proposed uses Multi-Objective Optimization (MOO) to construct the General Service overlay network (GSON) topology. With the MOO model, this algorithm has taken into account the reusing of nodes and links and matched the physical network. Visual topology decreases the cost of signal links and the overall network. A series of experimental simulation is designed to analyze the Algorithm performance. The results show that the algorithm has a better tradeoff in running time and performance than similar algorithms.
\end{abstract}

Keywords: Neural Network; Fuzzy theory; Fuzzy neural networks; QoS Evaluations.

\section{Introduction}

QoS (Quality of Service)is providing guaranteed quality-assured network services. QoS guarantees that the technology mainly known from two aspects control and management over network. Indicators are used to measure the QoS and set of parameters which are used in networks. It is used for enhancing user experience through network which includes: bandwidth, delay, jitter, packet loss, availability and throughput. To achieve the QoS guarantee, Business management system must provide efficient QoS Assessment methods to solve the current network status assessment for different services. Different business affected by different QoS indicators that they assess facing and considering a lots of problem which are made by different indicators. QoS assessment is closely related to the user experience that How to objectively reflect the user experience to judge the results. It is a problem worth studying [2, 3, 4, 11, 12].

Neural networks mimic the human brain theory of synaptic connections and information in nerve transmission. It has a strong learning ability and adaptive characteristics. It can learn the knowledge processing system having a black box features. You can achieve accurate simulation of complex systems. The Fuzzy Theory advantage depends upon its reasoning in line with the human thinking logic of habit $[9,14,13]$.

It does not need a lot of training data, but the reasoning process is determined by its membership function. Also there exists a strong subjectivity even with the expert system. Not only their subjective reasoning can be eliminated along with dynamic adjustment but also there is a problem to change the circumstances in the expert system. Therefore, flexibility fuzzy theory is relatively less usage and vulnerable for subjective human influence.

The neural network has strong learning ability. They can improve their training data sets by their methodology. Thus, it is more objective determining membership functions and inference rules. Basically, Fuzzy Neural Network (FNN) is developed on the basis of neural networks and fuzzy theory. It can make a fuzzy neural network in data processing deficiencies and flaws in fuzzy logic of learning. It's Give full play of advantages [1, 4, 5, 7, 16, 19, 13].

Neural networks represent membership functions and inference rules back piece. Analog meets user quality of service requirements in training data. Fuzzy neural network model has been driven after training and Generate simulation data results. The system gets QoS assessment with fuzzy neural network. [2, 6, 13]. 


\section{Related Work}

References the main focus of fuzzy theory is fuzzy reasoning and fuzzy comprehensive judgment. The results depend on fuzzy reasoning and Fuzzy comprehensive judgment method. The fuzzy comprehensive judgement is a qualitative concept which is used into the weighting factor and quantitative evaluation matrix representation in distribution. So, the matrix should be used for operation judgment. The implication depends upon fuzzy relation synthesis algorithms and fuzzy sets. Fuzzy system determines fuzzy implication. That is generally ok, but the law is not a unique for synthesis operation $[6,10]$.

An algorithm and technique has been proposed by Mr. Yingqi Zhang et al, Their paper basically concerned about the dissipativity analysis and design of discrete Markovian jumping neural networks with sector-bounded nonlinear activation functions and time varying delays. It is represented by Takagi-Sugeno fuzzy model. Some common condition is provided to guarantee that the augmented fuzzy jump neural networks are stochastically dissipative. After showing some valid examples, they prove the effectiveness and potential of the new design techniques by using fuzzy jump neural network. The criteria are formulated in terms of linear matrix inequalities [20].

An algorithm has been proposed by Mr. Yevgeniy Bodyanskiy et al. They proposed a new hybrid generalized additive wavelet-neuro fuzzy-system of computational intelligence and its learning algorithms. Their system has universal learning abilities which are related to the neural networks and neuro-fuzzy systems. Moreover, the system is the combination of neuro fuzzy system of TakagiSugeno-Kang and wavelet neural networks. They also generalized additive models of Hastie-Tibshirani. Their system has universal learning abilities which is related to the neural networks and neuro-fuzzy systems [21].

At present, the main focus of fuzzy theory and integration of neural networks is combination of the following three forms: FNN; Neuro fuzzy system (NFS); Fuzzy technology and neural network fuzzy neural hybrid system (NFHS). There are many kinds of fuzzy neural networks which includes fuzzy associative memory (AFM), fuzzy adaptive resonance theory (FART), Fuzzy multilayer Perception (FMLP). The focus of this paper is on three kinds of neural networks. These are FNN, NFS and NFHS $[1,2,6,13]$.

\section{Fuzzy Neural Network Model Construction}

A reasoning model is constructed in this paper which is a type of Fuzzy Neural Network as shown in Fig. 1. The input vector represents $\vec{x}=\left(x_{1}, x_{2}, \cdots x_{n}\right)^{T}$ a time series of monitoring the QoS indicators. $N N_{m f}$ (neural network membership functions) represents the input space which is mapped a degree of neural network membership function and described Calculation rules applicable degree of network. $N N_{1}, N N_{2}, \ldots \ldots ., N N_{m}$ represents the generation of regular structure of neural network, which is part of a single rule of inference rules. The neural networks are shown by numbers which they represent the calculation rules of applicable degree. Output Y represents the moment of business of QoS assessment.

The workflow of whole system is following a prescribed procedure: first input $\vec{x}$ according to determined m membership function in "if" part. $N N_{m f}$ Will work out normalized fitness $a_{1}, a_{2}, \cdots, a_{m}$ for each rule. When input $\vec{x}$ meanwhile by using neural networks $N N_{1}, N N_{2}, \cdots, N N_{m}$ to mimic $m$ "then" part generate the rule for in $\vec{x}$ and give the corresponding results $g_{1}, g_{2}, \cdots, g_{m}$. The final system output $y$ got by a certain way. This is shown in Fig.1 [8]. 


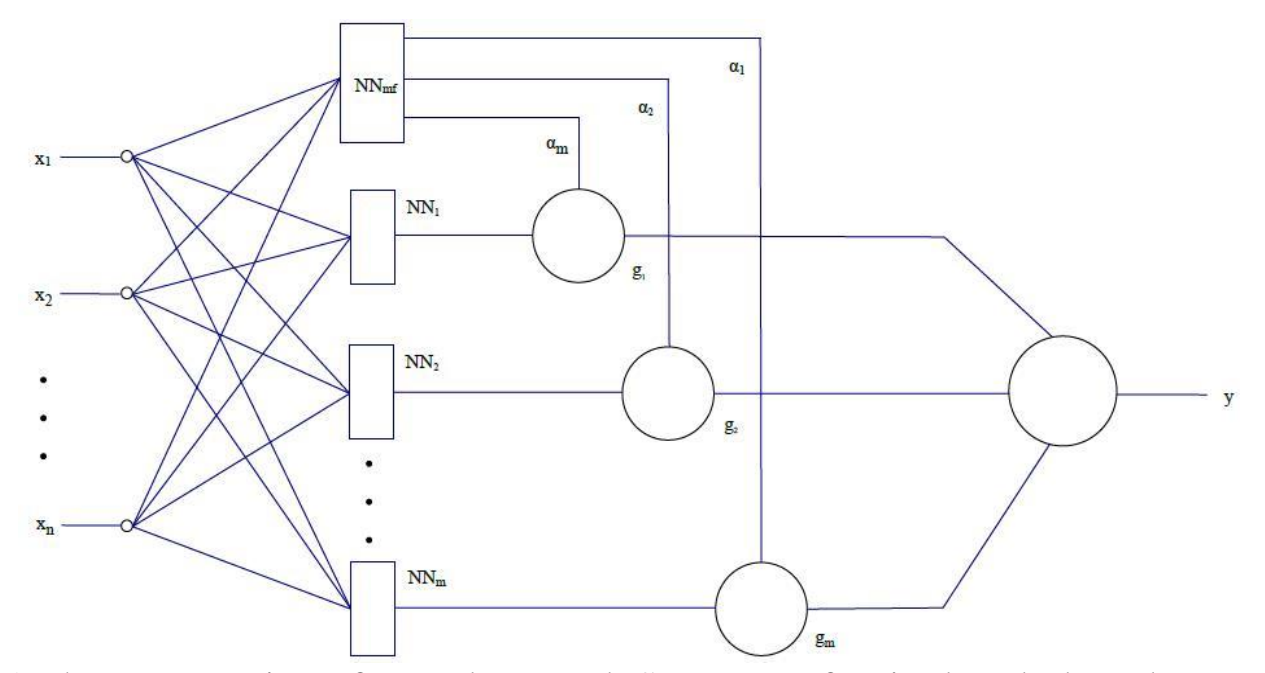

Fig. 1 The construction of neural network Structure of a single rule-based reasoning

$R_{j}$ : In case $\vec{x}=\left(x_{1}, x_{2}, \cdots x_{n}\right)^{T}$ for $A$. Then, $y_{j}=N N_{j}\left(x_{1}, x_{2}, \cdots, x_{n}\right)(j=1,2, \cdots, m)$

Where: $m$ is the number of inference rules, $A$ represents Inference rules of a former member of the fuzzy set $N N_{j}\left(x_{1}, x_{2}, \cdots, x_{n}\right)$ represent described neural network inference rules when input is $\vec{x}$ and output is $y_{j}$ through certain way.

\section{Algorithm Process}

The algorithm is divided into two phases, the first phase is to realize constraint satisfaction and meet the constraints requirements which are node and links capacity in network. Virtual link deletes the reuse virtual nodes. Due to that the physical link cost increases. This phase is called construction phase of algorithm. The second phase achieves optimization needs. Topology optimization has minimum condition in networks which are based on virtual links. Therefore, the algorithm is divided into two stages: topology construction and topology optimization.

The Construction of topology is deployed on the Overlay nodes which are based on the physical network link. The construction of virtual link is used to calculate the cost of each virtual node and also others node which are used in network to connect links. The link cost is calculated by using hops. It reuses link as a constraints. The value of the computation time is changed dynamically with decrease the number of nodes and links in network.

Another phase, Topology optimization is built to satisfy the minimal sub graph which are based on multiple branches. The minimum spanning tree is constructed for each sub graph. The virtual link of the spanning tree is obtained by GSON. In the topology construction stage, the sorting service node is set up to be all connected physical path between the server's nodes. The network layer topology selects the number of hops as a path of cost. It avoids service node to construct the virtual links between two virtual nodes. The path cost is marked as infinite and jumped out of calculation and the cost of the virtual link is added to a physical unit. The reuse link is counted and the physical path is selected at the same time. Therefore, the link value will change with the reuse of nodes and links.

Topology structure is more complex and there are many virtual links between nodes. It needs to be further optimized. Cut off the redundant link, it reduces the size of routing table entries and it is easily implemented for GSON routing. GSON routing needs to find positioning and resources of multicast universal service network function. The selection of Depth graph traversal or breadth graph traversal algorithm is started from the root of the topology. Which is constructed to give undirected graph from traversal algorithm? The graph obtains one or more branches but more than one branch is not connected in this topology. By Using prim and other greedy algorithms, to show the performance of minimum spanning tree method, here is need to obtain a tree or generate a forest. Each spanning tree has one connected component. 
The optimized model construction is based on minimizing the costs and time in network. It is simple structure and easy to use during the load of the application, it can effectively improve the business of user's QoS and QoE.

\section{Simulation Environment}

In this paper, GT-ITM (Georgia Tech Internetworking Topology Models) topology generator is generating a random graph plane test simulation. It is devoted to research and development for large-scale simulation on Internet and this was introduced by the Georgia Institute of Technology in 2000. A lot of study material data use this topology to generate simulation results. Random topology is used for large-scale simulation in networks. Its simulation can objectively verify by the general applicability of algorithm. When results were compared with similar algorithms then experimental simulation results were more credible as compare to other algorithms. [17, 22]

GT-ITM can generate a variety of flat random graphs as a model. The basic model is a pure random model. It has different edge probability functions. GT-ITM includes Waxman1, Waxman2 and (Doar-Leslie) variety of power law topology. In Waxman 1 model, there is an edge from node $u$ to $\mathrm{v}$ and this is probability.

$P(\mathrm{u}, \mathrm{v})=\alpha \times \exp (-\mathrm{d} /(\beta \cdot \mathrm{L}))$ Where $0<\alpha, \beta<=1, \mathrm{~d}$ is the Euclidean distance between nodes $\mathrm{u}, \mathrm{v}$. $\mathrm{L}$ is the maximum distance between the plane of any two nodes. When we are going to increase the model then the sides will become shorter. For longer hops $\beta, \beta$ Increase the figure to increase the proportion of the long side.

A node which is used by the simulator input file format must build the model and select the topology randomly. The purpose of this experiment is to show the validity and effectiveness of the algorithm. Due to this Effective topology we can minimize the cost of network time and also verify the node and time complexity of this algorithm. The service node determines the number and size of the effective path. The other node is connected to the service node and forwards this service node to the next node.

This article is designed for GSON topology construction algorithm. The nodes which have virtual link number can obtain normal circumstances. The other agreed performance parameters and the node degree is to show low performance due to the high degree of nodes in network. The network will must increase its maintenance overhead. The GSON topology construction algorithm can be constructed in addition to the physical layer topology. They need to control the time performance. So, that the service node can effectively transmit data $[2,3,17,12]$.

\subsection{Data Analysis}

During the simulation, The Algorithm is selected two Overlay topology construction algorithm which are based on minimum spanning tree algorithm named KMST and TKMST respectively. The Algorithm selects node degree and its time performance evaluation. Node degree reflects the logical link in the node number of paths. The node contains a degree of penetration. In general, it is lower than the average node degree. It embodies the balanced use of network resources. The time complexity of the algorithm is directly overhead of the algorithm. It reflects the value of the actual networking applications. The comparison of above three algorithms can be determined by their node degree, time complexity and performance analysis which are shown in Fig. 2. The Abscissa GT-ITM topology generate random parameters $\alpha . \alpha$ Represents the size of the values and the numbers of nodes which are proportional in the network. In Fig. 3. Abscissa is another parameter $\beta$. $\beta$ adds a node constantly and increase the length below. For Simulation results, this article will set some parameters according to need: $\mathrm{s}=0.3[3,5]$. 


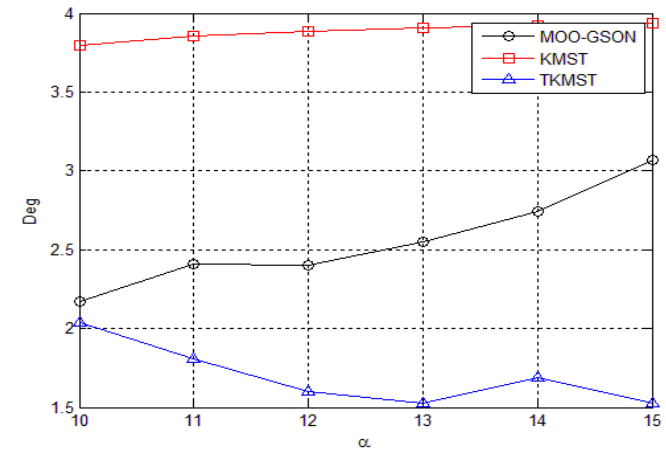

(A)Node degree

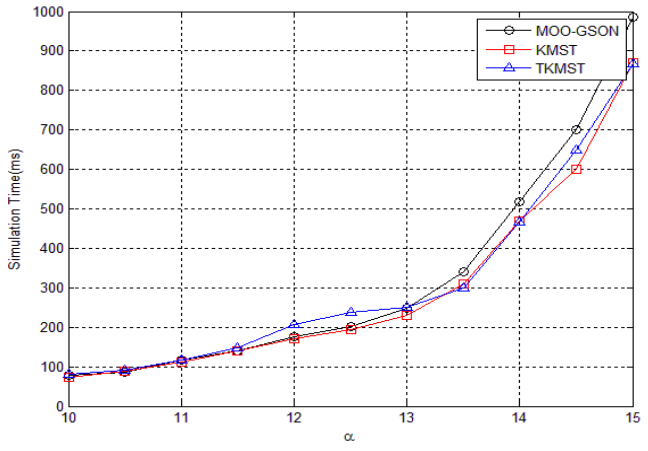

(B) Time complexity

Fig. 2 Three algorithms simulation performance Comparison Result 1.

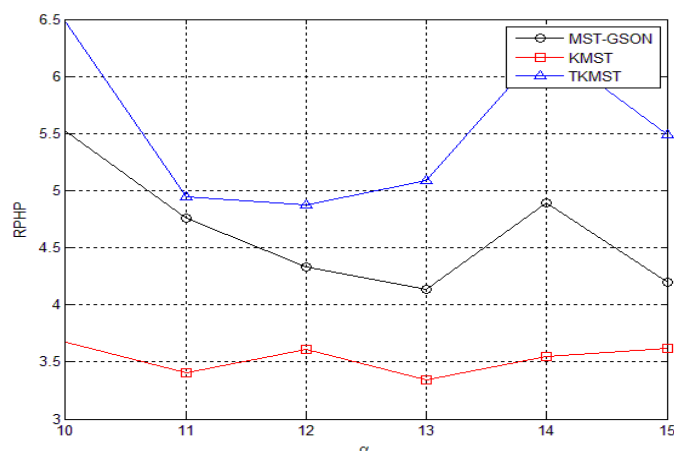

(C) Node Degree

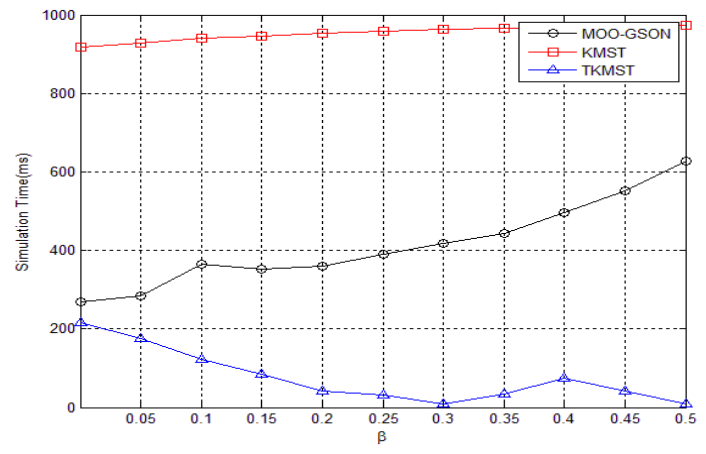

(D) Time Complexity

Fig. 3 Three algorithms simulation performance comparison Result 2.

Fig. 2, there is a schematic algorithms. A KMST algorithm and TKMST is the node algorithm. Which have simulation time, complexity and time comparison between the numbers of nodes. TKMST The average of the lowest node algorithm; the average node degree of the algorithm is in the intermediate position; while the average node degree KMST algorithm is much higher than the previous two algorithms. Mainly, because of the algorithm when the topology fully considers constructing reusable nodes, so the algorithm on a node is higher than KMST, less than TKMST. The time complexity of the algorithm increases when the node degree increases. The time complexity is increased between the two nodes, but due to increase in the number of nodes, the convergence rate is faster than others.

Fig.3, there is a schematic algorithm. A KMST algorithm and TKMST node algorithm. Which have simulation time, complexity and time comparison between the lengths of the side? It Increases the proportion of the long side within the same physical hops. This will reduce the link multiplexing in algorithm while the proportion of the long side shows no significant effect in the relationship between the three time complexities of the algorithm. The algorithm time complexity is still somewhere in between taking into account.

The success rate of construction this algorithm is depended on results. The simulation will have about 30\% probability KMST and TKMST algorithm. It cannot be complete overlay network construction mainly due to KMST, TKMST algorithms. To complete overlay network construction, a topology which is based on minimum spanning tree have encountered non-connected branches. By using this topology the algorithm result shows non normal convergence. This algorithm takes into account non-connected components. The topology optimization can be determined by non-connected components. So the algorithm is more practical as compare to others. 


\section{Conclusion}

Network virtualization is applied for physical network facilities. So without changing the network infrastructure, the network system is effectively enhance business for user through QoS and QoE. This paper focuses on Overlay topology construction problems. The multi-objective optimization method is used to model the problem and analyze the method for solving linear programming. It gives links and nodes to solve the reuse building basic scheme. The algorithm is based on the minimum spanning tree method. The original overlay topology optimization is a simple and efficient overlay topology. It reduces the overlay construction time and cost. It simplifies the server node router table space. In order to verify the effectiveness and practicality of the program, a brief comparison is made between theoretical analysis and simulation. Network simulation is using random generator which provides experimental data and compare it with traditional algorithms named KMST and TKMST. The optimization process increases the perception of nodes and convergence conditions. The results of the physical network topology are built a better Match. Experimental results show that the algorithm satisfies all constraints which are based on time and cost. Effectively, optimize the cost of generation.

\section{References}

[1] Yi C. Advances in Neural Networks - ISNN 2015 -12th International Symposium on Neural Networks, ISNN 2015, Jeju, South Korea, October 15-18, 2015, Proceedings [J]. 2015. 418-444.

[2] R Mijumbi, JL Gorricho, J Serrat, M Shen, K X - Mijumbi R, Gorricho J L, Serrat J, et al. A neuro-fuzzy approach to self-management of virtual network resources [J]. Expert Systems with Applications. An International Journal, 2015, 42 (3): 1376-1390.

[3] Barreiros M, Lundqvist P. QOS-Enabled Networks: Tools and Foundations [M]. Wiley Publishing, 2010. 3-33,

[4] Shi P, Zhang Y, Chadli M, et al. Mixed H-Infinity and Passive Filtering for Discrete Fuzzy Neural Networks With Stochastic Jumps and Time Delays [J]. IEEE Transactions on Neural Networks \& Learning Systems, 2015.

[5] Horikawa S I, Furuhashi T, Uchikawa Y. On fuzzy modeling using fuzzy neural networks with the back-propagation algorithm [J]. IEEE Transactions on Neural Networks, 1992, 3 (5): 801-806.

[6] Tsoukalas L H, Uhrig R E. Fuzzy and Neural Approaches in Engineering [M]. 1997

[7] Haykin S, Network N. A comprehensive Foundation [J]. Neural Networks, 2004.

[8] Dai H, Hua Q U, Zhao J. QoS Routing Algorithm with Multi-Dimensions for Overlay Networks [J]. Wireless Communication over Zigbee for Automotive Inclination Measurement China Communications, 2013, 10 (10):167-17

[9] Hamba K, Flateland I, Ystehede A. Joint 9th IFSA World Congress and 20th NAFIPS International Conference (IFSA/NAFIPS 2001) [J]. Systems Control \& Information, 2001, 45(6): $119-122$

[10]Zaiden L A. Fuzzy Logic [J]. Computer, 1988, 21(4): 83-93.

[11]Bardsiri A K, Hashemi S M. QoS Metrics for Cloud Computing Services Evaluation [J]. International Journal of Intelligent Systems Technologies \& Applications, 2014, 6 (12): 27-33.

[12] Yang R, Chen Q, Qi L, et al. A QoS Evaluation Method for Personalized Service Requests [C]// International Conference on Web Information Systems and Mining. Springer-Verlag, 2011: 393-402. 
[13]Mijumbi R, Gorricho J L, Serrat J, ET al.neuro Fuzzy approach to self-management of virtual network resources [J]. Expert Systems with Applications an International Journal, 2015, 42 (3): 1376-1390.

[14]Information on: https://nl.wikipedia.org/wiki/Fuzzy_logic1

[15]Information on: https://en.wikipedia.org/wiki/Inference

[16]Information on: https://en.wikipedia.org/wiki/Neuro-fuzzy

[17]Information on: http://www.isi.edu/nsnam/ns/nstopogen.html

[18]Information on: https://en.wikipedia.org/wiki/Rule_of_inference.

[19]N Information on: https://en.wikipedia.org/wiki/Nervous system

[20]Zhang, Yingqi, et al. "Dissipativity Analysis for Discrete Time-Delay Fuzzy Neural Networks with Markovian Jumps." IEEE Transactions on Fuzzy Systems 24.2 (2016): 432-443. 